\title{
Measurement of Capacitive Accelerometer with Decreased Nonlinearity of Static Characteristic
}

\author{
S. Timoshenkov, V. Kalugin, A. Shalimov, A. Timoshenkov, M. Golovinskiy, S. \\ Berezueva \\ National Research University of Electronic Technology \\ Russian Federation
}

\begin{abstract}
A series of the experimental works, which allowed achieving an evident improvement of functional parameters of MEMS accelerometers, was performed by specialists of the Microelectronics Department MIET. In particular - decrease the nonlinearity of the static characteristic. This article describes methods, which allowed achieving these results.
\end{abstract}

\section{Keywords-MEMS accelerometer; \\ capacitive} accelerometer; capacitance-to-voltage converter

\section{INTRODUCTION}

The Colibrys company is one of the conventional world leaders in the field of production of MEMS-accelerometers which releases accelerometers on various ranges of the measured accelerations. The nonlinearity declared by this company for range $\pm 5 \mathrm{~g}$ is $<0.8 \%$ (MS9005). The performed researches showed that our department is capable to provide values of nonlinearity of the static characteristic of $<0.1 \%$ (for ranges, including $\pm 5 g$ ).

\section{IIDEVELOPMENT AND MEASUREMENT OF CAPACITIVE ACCELEROMETER}

Deep etching silicon elements of MEMS capacitive accelerometers was carried out on I-Speeder (Alcatel). We used silicon wafers by thickness $300 \mathrm{mkm}$ with orientation of surfaces of wafer (100) [1, 2]. Bonding silicon with Boroflat33 glass was carried out on SB6 Carl Suss (Fig. 1). For measurement considered parameters we used different climatic (Espec Corp MC-811P) and mechanical (LDS V455 with PA 1000L and Acutronic 1135S) devices.

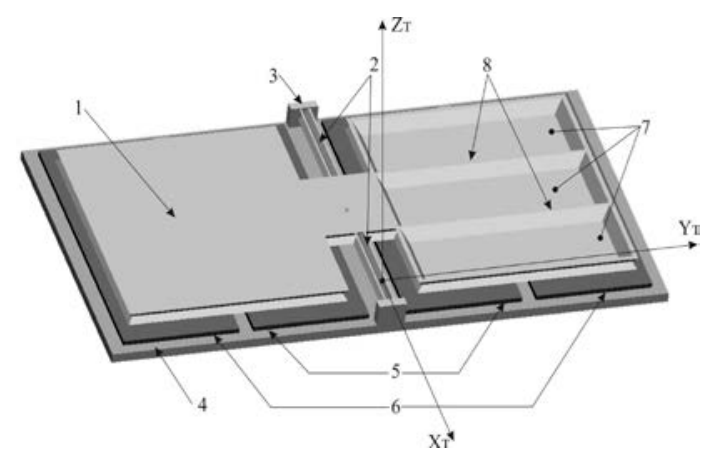

FIGURE I. SENSOR ELEMENT OF A CAPACITIVE ACCELEROMETER.

1 - inertial mass;

2 - torsions;

3 - silicon substrate;

4 - glass stator with two pairs of electrodes;

5 - electrode capacitive system removal movements;

\section{6 - electrode electrostatic torque sensor}

Important parameter of accelerometer - providing highspeed performance of the device-frequency range of the transformation. Comparing to various samples of Colibrys, it is possible to make a conclusion that production of this company allows to reach values $>3000 \mathrm{~Hz}$ at nonlinearity of $<1 \%$. The similar result is given by the analysis of production of the company Analog Devices: at frequency range of $2.26 \mathrm{kHz}$ nonlinearity makes from (0.5 to 2.5$) \%$ (ADIS16006). Our production allows reaching the values of frequency range up to $1000 \mathrm{~Hz}$ (including measurement of static accelerations) with nonlinearity less than $0.1 \%$. This result was achieved according to utilization of two solutions: improved construction of sensing element and scheme of processing of signal. Speaking about scheme it is necessary to say, that it is very difficult to achieve low nonlinearity of function describing transformation of acceleration to capacitance, which performs sensing element. It can be explained due to physics of transformation in pendulum construction [3].

Dependence between applied acceleration a and angle of rotation of moving mass $\alpha$ can be represented as follows:

$\alpha=e^{-\xi \omega_{0} a}\left(\cos \left(\omega_{0} a \sqrt{4-\xi^{2}}\right)+\sin \left(\omega_{0} a \sqrt{4-\xi^{2}}\right)\right)+\frac{m l}{I} \cdot \frac{a-2 \xi}{\omega_{0}^{2}}(1)$

Where:

$\alpha$ - angle of rotation of moving mass;

$\xi$ - damping ratio;

$\omega_{0}$ - natural frequency of the sensing element;

$I$ - moment of inertia;

$l$ - distance between the mass center and the center of pad on the stator;

\section{$m$ - mass of the rotor.}

Otherwise, if scheme performs only forward transformation of capacitance to output signal, relatively high nonlinearity occurs. Other method is to apply the output signal back to sensing element i.e. organize feedback which allows to decrease the influence of sensing element to the parameters of the accelerometer on the whole. It can be achieved because of the following: if feedback will be organized as a negative feedback, the electrostatic force, applied to the sensing element will return the rotor to the initial position so it will not 
influence on the device or this influence will be significantly decreased. Theoretically, this solution was described many times in literature as a PID-regulator. But, at the same time, everywhere was mentioned that it is very difficult to achieve the optimal parameters of such regulator because of the influence of different factors. First and the main problem is that the transfer function of sensing element can be described only as an oscillator function. But in this situation it is much more difficult to calculate coefficients of PID-regulator in comparison with the aperiodic or critical transfer functions. Our specialist performed a range of experiments with sensing element and then - with the help of Ansys (for construction modeling) and OrCAD (for Pspice modeling) - get the model of accelerometer with PI-regulator (Fig. 2).

This scheme works as follows: excitation of sensing element represented as a pulse generator with fixed frequency. The initial value of amplitude is formed by voltage reference, which with the help of inverting and non-inverting amplifiers forms the positive and negative amplitude. So, the amplitude of the signal from the middle point of the sensing element corresponds to the value of disbalance of its capacitances. Therefore, it corresponds to the applied acceleration. Then this signal transforms to the output voltage with the help of capacitance-to-voltage converter and output filter. On the other hand, capacitance-to-voltage converter forms the negative feedback, which corrects the amplitude of excitation signal, therefore decreasing the influence of sensing element on the whole device.

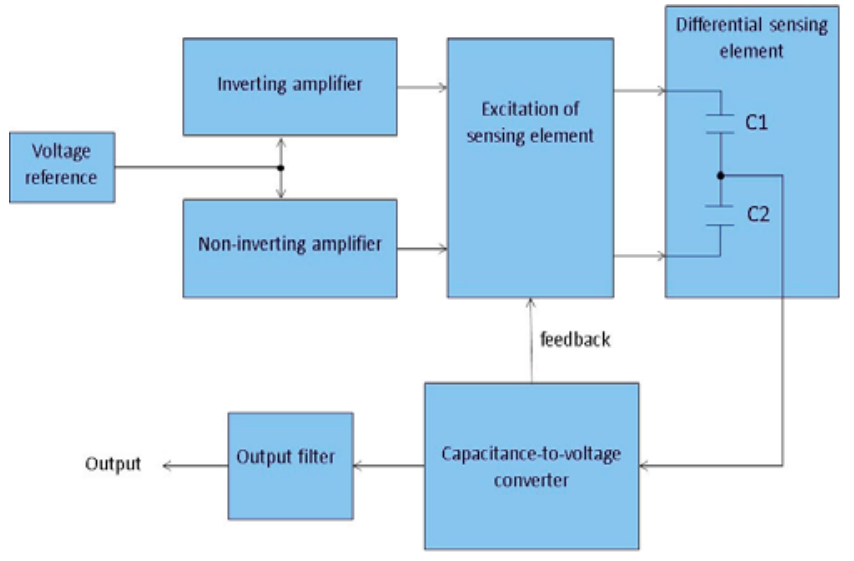

FIGURE II. FLOW-CHART OF CAPACITIVE ACCELEROMETER WITH PI-REGULATOR.

Certainly, it doesn't mean that we will end our investigations, this only means, that we made the next step to the accelerometer with the best parameters. This conclusion confirms with the results of testing our samples, which showed that it was achieved undoubted improvement of parameters. Also, it is necessary to mention, that in addition to main parameters accelerometer have to own a range of other also very important - parameters, describing the availability of sensor to work under stress factors.

Speaking about resistance to stress factors, it is necessary to say that our specialists performed tests of functioning sensors for different ranges of the measured accelerations in the mode of fast change of temperatures with the increased or lowered values of power supply (Fig. 3). On this figure you can see, that temperature changes very rapidly - as rapidly, as allows climatic camera. And the range of this changing is rather wide - from minus 60 to plus $85^{\circ} \mathrm{C}$. At the same time, the power supply changes from higher $( \pm 5.25 \mathrm{~V})$ to lower $( \pm 4.75 \mathrm{~V})$ thus providing two stress factors at the same time. The results of measuring of the output signal showed with the blue line allows us to say that during of the whole experiment the sample demonstrates the resistance to described stress factors.

Other test confirming excellent functional characteristics of our accelerometers was carried out in the following mode: the rotary stand continuously turned a sample with a speed of $30 \%$ sec, changing, thus, the size of a projection of the influencing acceleration in the range $\pm 1 \mathrm{~g}$. As a result at the exit of the transducer was observed the voltage signal of a sinusoidal form proportional to acceleration (Fig. 4). The heat chamber changed temperature in the range from $(-20$ to +85$) \mathrm{C}$. This experiment gives us the possibility to claim about high temperature stability of scale factor of our accelerometer.

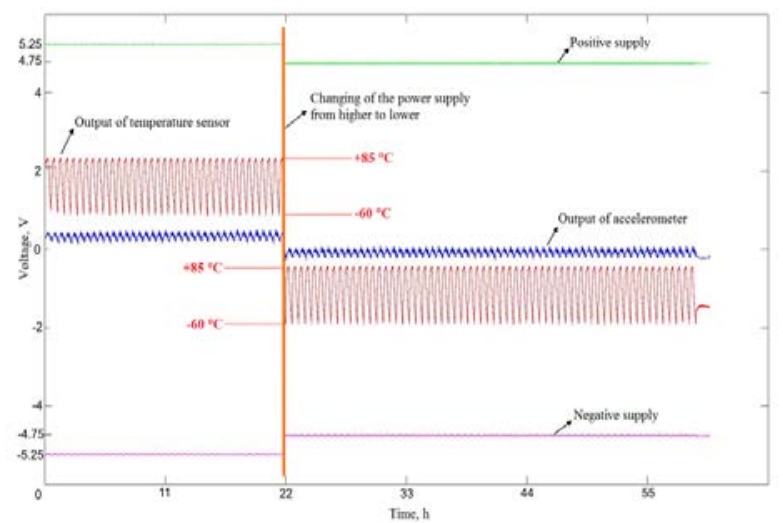

FIGURE III. THE TEMPORARY CHART OF TEST ACCELEROMETER RESISTANCE TO THE RIGID MODES OF OPERATION (ON THE EXAMPLE SAMPLE FUNCTIONED IN RANGE $\pm 1.2 \mathrm{G}$ ).

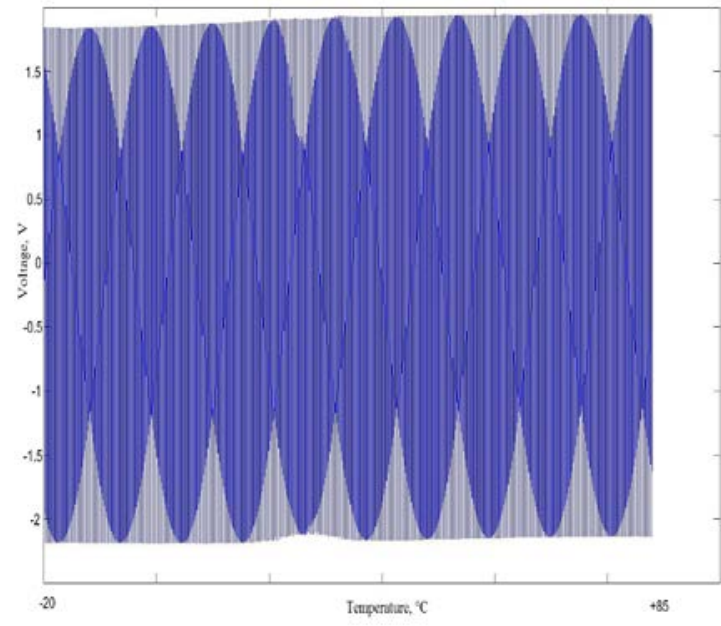

FIGURE IV. TEMPERATURE DRIFT OF SCALE FACTOR OF MEMS ACCELEROMETER. 


\section{SUMMARY}

The current work describes the solution, which allows to significantly decreasing one of the most important parameters of MEMS accelerometers, in particular, nonlinearity of static characteristic. Also, it was shown that described solution allows achieving the resistance of the device to several stress factors. The results of measurement are also presented.

\section{REFERENCES}

[1] N. Lobontiu, E., Garcia, Mechanics of Microelectromechanical Systems, Springer, Kluwer academic publishers, Boston, 2005, pp. 263-286.

[2] S. Beeby, G. Ensell, M. Kraft, N. White, MEMS Mechanical Sensors, Artech House. Inc., Boston, London, 2004. pp. 4-94.

[3] E. Gaura, R. Newman, M. Kraft, A. Flewitt, D. William, L. Monteiro, Smart MEMS and Sensor Systems, Imperial College Press, London, 2006, pp. 233-271. 